\title{
Production spectra of zero-degree neutral particles measured by the LHCf experiment
}

\author{
A. Tiberio ${ }^{1,2, a}$, on behalf of the LHCf Collaboration \\ ${ }^{1}$ University of Florence, Italy \\ ${ }^{2}$ INFN Section of Florence, Italy
}

\begin{abstract}
The LHC-forward (LHCf) experiment, situated at the LHC accelerator, has measured neutral particles production in a very forward region (pseudo-rapidity $\eta>8.4$ ) in proton-proton and proton-lead collisions. The main purpose of the LHCf experiment is to test hadronic interaction models used in ground based cosmic rays experiments to simulate cosmic rays induced air-showers in the Earth's atmosphere.

The experiment is composed of two independent detectors located at $140 \mathrm{~m}$ from the ATLAS interaction point (IP1) on opposite sides; each detector is composed of two sampling and position sensitive calorimeters.

In this paper, latest published physics results from $\mathrm{p}-\mathrm{p}$ and $\mathrm{p}-\mathrm{Pb}$ collisions (at $\sqrt{s}=7$, $2.76 \mathrm{TeV}$ and $\sqrt{s_{N N}}=5.02 \mathrm{TeV}$, respectively) compared with Monte Carlo predictions of DPMJET, EPOS, PYTHIA, QGSJET and SIBYLL event generators will be presented. In particular, the inclusive energy spectra of neutrons in p-p collisions and the transverse and longitudinal momentum spectra of neutral pions for different pseudo-rapidity ranges in $\mathrm{p}-\mathrm{p}$ and $\mathrm{p}-\mathrm{Pb}$ collisions will be shown; then, test of Feynman scaling hypothesis using neutral pion spectra will be discussed. Preliminary results of photon inclusive energy spectra in p-p collisions at $\sqrt{s}=13 \mathrm{TeV}$ will be also presented.
\end{abstract}

\section{Introduction}

The LHC-forward experiment (LHCf) has measured neutral particles production in a very forward region in proton-proton and proton-lead collisions at the Large Hadron Collider. The main purpose of LHCf is to improve hadronic interaction models of Monte Carlo (MC) simulations used in cosmic rays indirect measurements. Highest energy cosmic rays can only be detected from secondary particles which are produced in the interaction of the primary particle with nuclei of the atmosphere, the socalled air showers. Studying the development of air showers, it is possible to reconstruct the type and kinematic parameters of primary particles. To reproduce the development of air showers, MC simulations with accurate hadronic interaction models are needed. Since the energy flow of secondary particles is concentrated in the forward direction, measurements of particle production in the high pseudo-rapidity region (i.e. small angles) are very important. Furthermore, in the forward region soft QCD interactions (non perturbative regime) dominates and MC simulations of air showers are based on phenomenological models, so inputs from experimental data are crucial for the tuning of that

\footnotetext{
ae-mail: alessio.tiberio@fi.infn.it
} 
models. LHC accelerator gives the possibility to study a wide range of collision energies, from 0.9 $\mathrm{TeV}$ to $13 \mathrm{TeV}$ in the center of mass frame, which corresponds to an energy range in the laboratory frame from $10^{14} \mathrm{eV}$ to $0.9 \times 10^{17} \mathrm{eV}$. This energy range covers the "knee" region of cosmic rays spectrum, which occurs around $10^{15} \mathrm{eV}$.

\section{The detector}

LHCf is composed of two independent detectors, called Arm1 and Arm2. Arm1 is located 140 meters away from ATLAS interaction point (IP1) in the IP8 direction, while Arm2 is placed 140 meters away from IP1 in the opposite direction (toward IP2). Detectors are placed inside Target Neutral Absorber (TAN), where the beam pipe turns into two separates tubes. Since charged particles are deviated by the D1 dipole magnet (which bends colliding beams into the two separate beam pipes), only neutral particles, mainly photons and neutrons, reach the detector.

Each detector was made of two sampling and imaging calorimeters (called towers hereafter). Each tower is composed of 16 tungsten layers and 16 scintillator layers to measure the energy deposit and it also contains 4 position sensitive layers. During $0.9 \mathrm{TeV}, 2.76 \mathrm{TeV}$ and $7 \mathrm{TeV}$ operations at the LHC, plastic scintillators (EJ-260) were used. Arm1 detector used scintillating fiber ( $\mathrm{SciFi}$ ) to measure position, while Arm 2 used silicon microstrip detectors. For $13 \mathrm{TeV}$ operation both detectors were upgraded: all the plastic scintillators were replaced by $\mathrm{Gd}_{2} \mathrm{SiO}_{5}$ (GSO) scintillators because of their radiation hardness; also the Arm1 SciFi were replaced by GSO bars. In Arm2 the signal of silicon detectors was reduced using a new bonding scheme of the microstrips to avoid saturation of readout electronics due to the higher energy deposit expected at $\sqrt{s}=13 \mathrm{TeV}$.

Transverse cross sections of towers are $20 \times 20 \mathrm{~mm}^{2}$ and $40 \times 40 \mathrm{~mm}^{2}$ for Arm 1 and $25 \times 25 \mathrm{~mm}^{2}$ and $32 \times 32 \mathrm{~mm}^{2}$ for Arm2. Longitudinal dimension of towers is of 44 radiation lengths, which correspond to 1.6 nuclear interaction lengths. Energy resolution is better than $5 \%$ for photons and of about $40 \%$ for neutrons. Position resolution for photons is $200 \mu \mathrm{m}$ and $40 \mu \mathrm{m}$ for Arm1 and Arm2 respectively, while position resolution for neutrons is of about $1 \mathrm{~mm}$. Smaller tower of each detector is placed on the beam center and covers the pseudo-rapidity range $\eta>9.6$, while larger tower covers the pseudo-rapidity range $8.4<\eta<9.4$. More detailed descriptions of detector performance are reported elsewhere [1-3].

\section{Experimental results}

LHCf collected data in three different low-luminosity dedicated runs in 2010, 2013 and 2015. Protonproton collisions at $\sqrt{s}=900 \mathrm{GeV}(2010), \sqrt{s}=2.76 \mathrm{TeV}$ (2013), $\sqrt{s}=7 \mathrm{TeV}$ (2010) and $\sqrt{s}=13$ $\mathrm{TeV}$ (2015) were produced; proton-lead collisions at $\sqrt{s_{N N}}=5.02 \mathrm{TeV}$ were produced in 2013 .

Results for single photon energy spectra in p-p collisions at $\sqrt{s}=900 \mathrm{GeV}, 7 \mathrm{TeV}$ and for $\pi^{0}$ transverse momentum spectra in p-p and p-Pb collisions at $\sqrt{s}=7 \mathrm{TeV}$ and $\sqrt{s_{N N}}=5.02 \mathrm{TeV}$, respectively, have already been published [4-7]. Latest published results are presented in this paper: neutron energy spectra in p-p collisions at $\sqrt{s}=7 \mathrm{TeV}$ [8] are shown in Sect. 3.1 while results from $\pi^{0}$ transverse $\left(P_{T}\right)$ and longitudinal $\left(P_{Z}\right)$ momentum analysis in $\mathrm{p}-\mathrm{p}$ at $\sqrt{s}=2.76,7 \mathrm{TeV}$ and $\mathrm{p}-\mathrm{Pb}$ collisions at $\sqrt{S_{N N}}=5.02 \mathrm{TeV}$ [9] are shown in Sect. 3.2. Finally, also preliminary result from photon analysis at $\sqrt{s}=13 \mathrm{TeV}$ will be presented in Sect. 3.3.

\subsection{Neutron energy spectra in p-p collisions}

In this section results from neutron analysis in p-p collisions at $\sqrt{s}=7 \mathrm{TeV}$ are presented [8]. Data were taken during LHC Fill \#1104 on May 15, 2010. Integrated luminosities were $0.68 \mathrm{nb}^{-1}$ and 0.53 
$\mathrm{nb}^{-1}$ for Arm1 and Arm2 respectively, with a typical luminosity of $6.4 \times 10^{28} \mathrm{~cm}^{-2} \mathrm{~s}^{-1}$, derived from LHCf front counters [10].

Due to the limited dimensions of calorimeter, hadronic showers are not fully contained in the tower and the study of hadronic neutral particles is less precise and more complicated than photons. Hadronic particles (mostly neutrons) are selected using information of longitudinal distribution of energy deposit in the calorimeter. Since the energy resolution for neutrons is $\sim 40 \%$, the true energy spectra are smeared by the detector response. Smeared energy spectra of neutrons measured by Arm 1 and compared with MC simulation predictions of several models are shown in figure 1 in three pseudorapidity ranges.
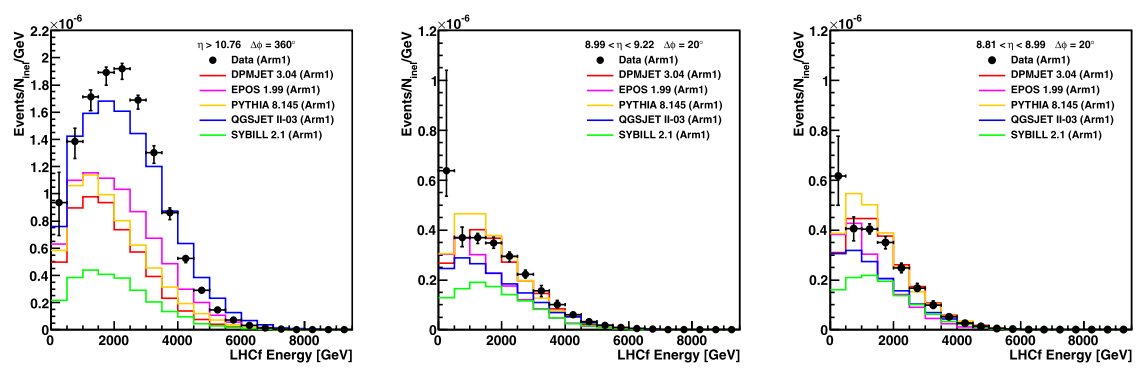

Figure 1. Energy distribution of neutrons in p-p collisions at $7 \mathrm{TeV}$ before unfolding in three pseudo-rapidity regions: coloured histograms show results from Monte Carlo simulations with different hadronic interaction models, black points show experimental data of Arm1. Particles above maximum available energy ( $3500 \mathrm{GeV})$ are clearly due to the smearing effect of the detector.

Unfolding technique is therefore needed to estimate the true energy spectra: a multidimensionalspectra unfolding Bayesian method [11] relying on energy and $P_{T}$ measured variables was used. Results after unfolding are shown in figure 2. It is evident from the results that in the very forward pseudo-rapidity region $(\eta>10.76)$ there is a large amount of high energy neutrons, only predicted by the QGSJETII-03 [12] model. In other regions data lie between models predictions, but there is not an unique model that represents data.
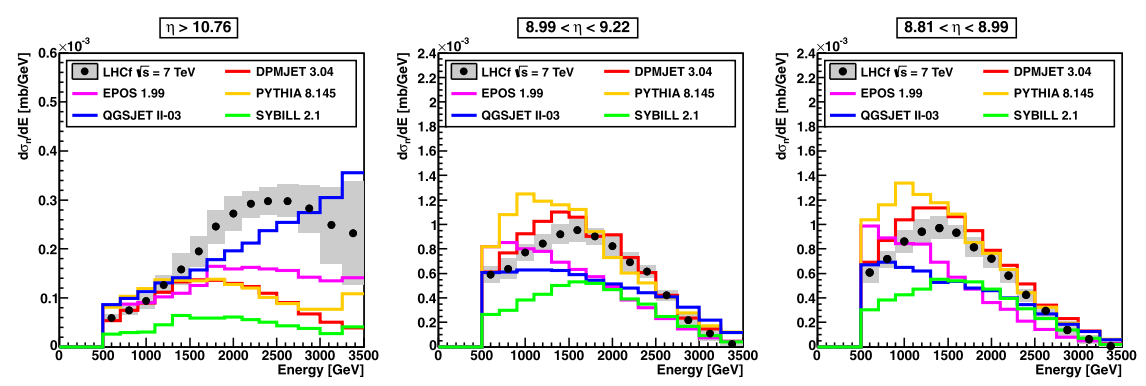

Figure 2. Energy distribution of neutrons in p-p collisions at $7 \mathrm{TeV}$ after unfolding in three pseudo-rapidity regions: coloured histograms show results from Monte Carlo simulations with different hadronic interaction models, black points show combined experimental data of Arm1 and Arm2. A large amount of very-forward $(\eta>10.76)$ high energy neutrons is predicted by QGSJETII- 03 and confirmed by data. 


\subsection{Neutral pion $P_{T}$ and $P_{Z}$ spectra in $\mathrm{p}-\mathrm{p}$ and $\mathrm{p}-\mathrm{Pb}$ collisions}

Published results [9] for $\pi^{0}$ transverse and longitudinal momentum spectra in proton-proton collisions at $\sqrt{s}=7 \mathrm{TeV}$ will be shown in Sect. 3.2.1. Results from comparison with proton-lead collisions data collected at $\sqrt{s_{N N}}=5.02 \mathrm{TeV}$ will be presented in Sect. 3.2.2. A test for the Feynman scaling rule will be shown in Sect. 3.2.3.

In previous published results $[6,7], \pi^{0} \mathrm{~s}$ were reconstructed from energy and incident position of their decay photons in events with one incident photon in each tower ("Type I" event), while events with two incident photons in the same tower ("Type II" event) were rejected. In this analysis Type II events were included for the first time. The inclusion of Type II events allowed to extend the coverage in the $P_{T}-P_{Z}$ phase space as shown in figure 3.

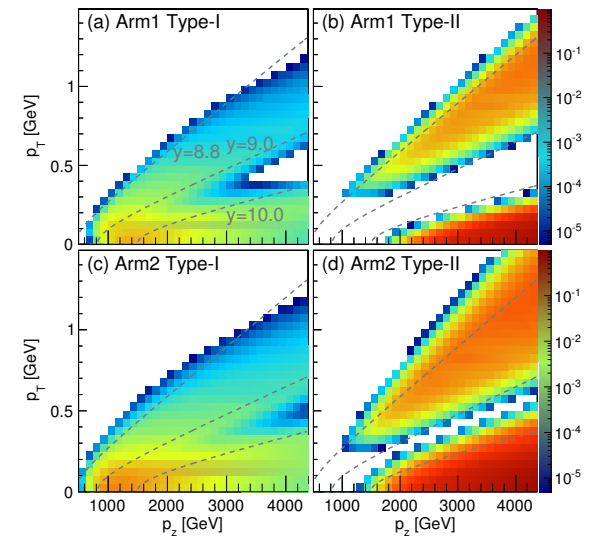

Figure 3. Acceptance map of $\pi^{0}$ s detected by LHCf in $P_{T}-P_{Z}$ phase space for Arm1 (top figures) and Arm2 (bottom figures). Type II $\pi^{0}$ s (right figures) extended the phase space coverage up to $P_{T} \sim 1 \mathrm{GeV}$, while with only Type I events the maximum detectable $P_{T}$ was $\sim 0.5 \mathrm{GeV}$.

\subsection{1 $P_{T}$ and $P_{Z}$ distributions at $\sqrt{s}=7 \mathrm{TeV}$}

The data in p-p collision at $\sqrt{s}=7 \mathrm{TeV}$ were obtained during LHC Fills \#1104, \#1107, \#1112 and \#1117 from May 15 to 22, 2010. The integrated luminosity was $2.67 \mathrm{nb}^{-1}$ for Arm 1 and $2.10 \mathrm{nb}^{-1}$ for Arm2.

Measured $P_{T}$ spectra are compared with predictions from different MC interaction models in several regions of the rapidity $y$, as shown in figure 4. QGSJET II-04 model [12] shows a good overall agreement with LHCf data, while EPOS-LHC [13] has a good agreement at low $P_{T}(<0.5 \mathrm{GeV})$ and shows a slightly harder distribution for $P_{T}>0.5 \mathrm{GeV}$. Both models are based on the parton-based Gribov-Regge approach $[14,15]$ and they are tuned using ALICE, ATLAS, CMS and TOTEM data. The prediction of SIBYLL 2.1 [16] agrees well with the data for $8.8<y<9.2$ and $P_{T}<0.4 \mathrm{GeV}$ while its absolute yield is about half that of the data for $y>9.2$. The predictions of DPMJET 3.06 [17] and PYTHIA $8.185[18,19]$ are compatible with data for $9.0<y<9.8$ and $P_{T}<0.2 \mathrm{GeV}$, while for $P_{T}>0.2 \mathrm{GeV}$ they become significantly harder than both LHCf data and the predictions of other models.

$P_{Z}$ spectra in several $P_{T}$ intervals are presented and compared with models predictions in figure 5 . There is again an overall agreement between LHCf data and the QGSJET prediction, especially for 0.0 $<P_{T}<0.2 \mathrm{GeV}$. The EPOS prediction is compatible with data for $P_{Z}<2 \mathrm{TeV}$, while showing a hard slope for $P_{Z}>2 \mathrm{TeV}$ in all $P_{T}$ regions. The predictions by DPMJET and PYTHIA agree with data for $P_{T}<0.2 \mathrm{GeV}$ and $P_{Z}<1.6 \mathrm{TeV}$, while showing a harder distribution for the higher $P_{Z}$ regions. SIBYLL predicts a smaller production of $\pi^{0} \mathrm{~s}$ for $P_{T}<0.2 \mathrm{GeV}$ and shows a similar behaviour with DPMJET and PYTHIA with increasing $P_{T}$. 


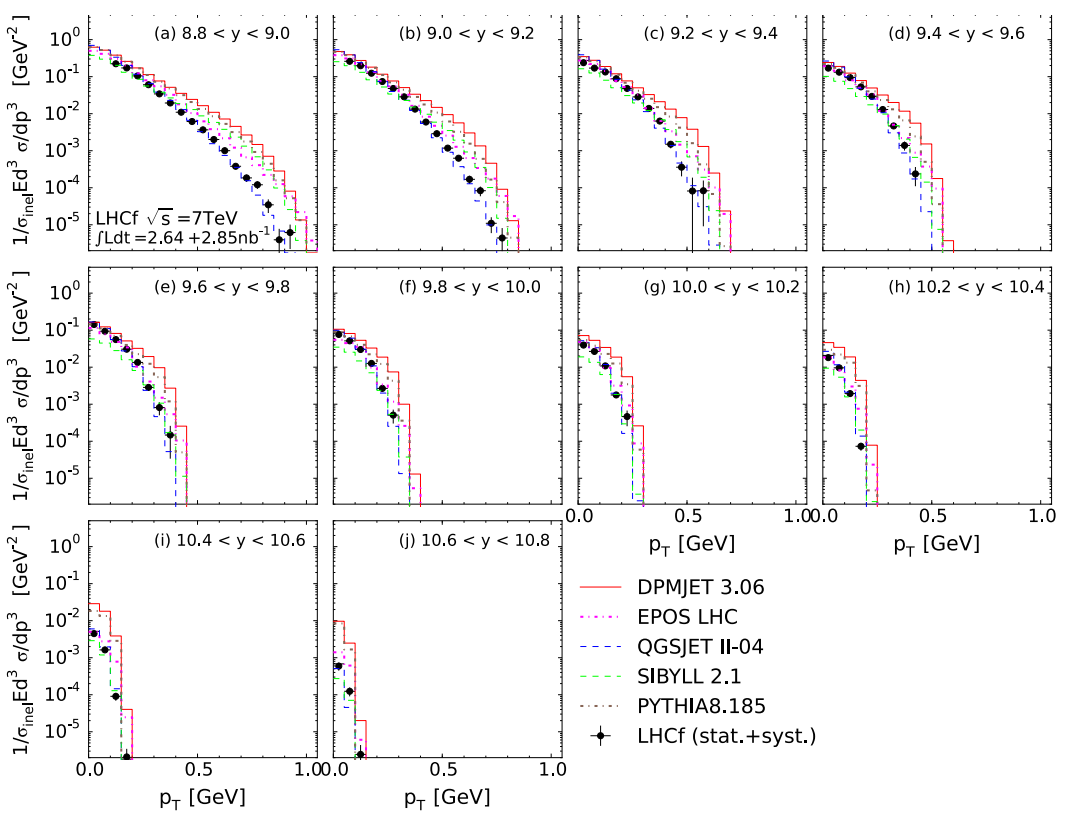

Figure 4. Transverse momentum distribution of neutral pions in several rapidity regions at $\sqrt{s}=7 \mathrm{TeV}$ : coloured histograms show results from Monte Carlo simulations with different hadronic interaction models, black points show experimental data.
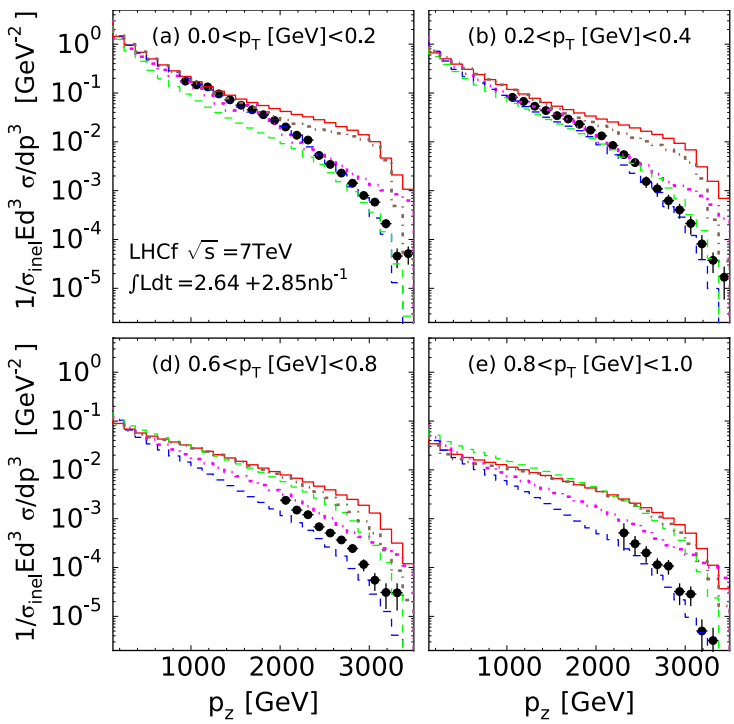

(e) $0.8<p_{\mathrm{T}}[\mathrm{GeV}]<1.0$

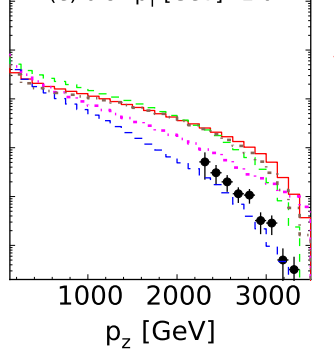

(c) $0.4<\mathrm{p}_{\mathrm{T}}[\mathrm{GeV}]<0.6$

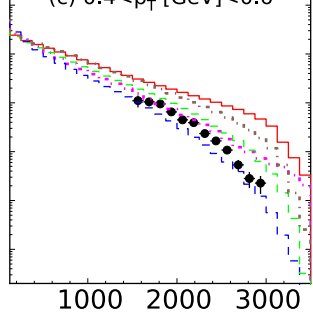

$\mathrm{p}_{\mathrm{z}}[\mathrm{GeV}]$

DPMJET 3.06

EPOS LHC

-.. - QGSJET II-04

SIBYLL 2.1

PYTHIA8.185

$\downarrow \quad$ LHCf (stat. +syst.)
Figure 5. Longitudinal momentum distribution of neutral pions in several $P_{T}$ regions at $\sqrt{s}=7 \mathrm{TeV}$ : coloured histograms show results from Monte Carlo simulations with different hadronic interaction models, black points show experimental data.

\subsubsection{Nuclear modification factor}

During the p-Pb run in 2013, only Arm2 detector was installed because of two different reasons: to minimize interference with ATLAS ZDC operation and because MC simulations predicted too high multiplicity of neutrons in $\mathrm{Pb}$-remnant side. As a consequence, Arm2 were installed on p-remnant side for almost all the time. Data recorded on p-remnant side in LHC Fills \#3478 and \#3481 on January 21-22, 2013 were used in the analysis, with an integrated luminosities of $0.63 \mathrm{nb}^{-1}$.

Proton-lead collisions are very important to understand nuclear effects on forward particles production, because the first collision of an air-shower happens between the primary cosmic ray (a proton 
or a nucleus) and a nucleus of the atmosphere. Comparing spectra in $\mathrm{p}-\mathrm{p}$ and $\mathrm{p}-\mathrm{Pb}$ collisions it is possible to estimate nuclear effects; in particular it is possible to measure the nuclear modification factor $\left(R_{p P b}\right)$ which is defined as

$$
R_{p P b} \equiv \frac{\sigma_{\text {inel }}^{p p}}{\left\langle N_{\text {coll }}\right\rangle \sigma_{\text {inel }}^{p P b}} \frac{E d^{3} \sigma^{p P b} / d p^{3}}{E d^{3} \sigma^{p p} / d p^{3}}
$$

where $E d^{3} \sigma^{p p} / d p^{3}$ and $E d^{3} \sigma^{p P b} / d p^{3}$ are the inclusive cross sections of $\pi^{0}$ production in p-p and $\mathrm{p}-\mathrm{Pb}$ collisions at $5.02 \mathrm{TeV}$ respectively, and $\left\langle N_{\text {coll }}\right\rangle$ is the mean number of binary nucleon-nucleon collisions. $\left\langle N_{\text {coll }}\right\rangle$ is estimated as 6.9 from MC simulations. Since there are no data in p-p collisions at $\sqrt{s}=5.02 \mathrm{TeV}$, the distribution $E d^{3} \sigma^{p p} / d p^{3}$ is obtained interpolating results at $7 \mathrm{TeV}$ and 2.76 $\mathrm{TeV}$ in p-p collisions. Nuclear modification factor as a function of $P_{T}$ in different rapidity regions is shown in figure 6. MC models predict a strong suppression $\left(R_{p P b} \sim 0.1\right)$ over all range of $P_{T}$ and it is consistent with the LHCf data within the uncertainties.

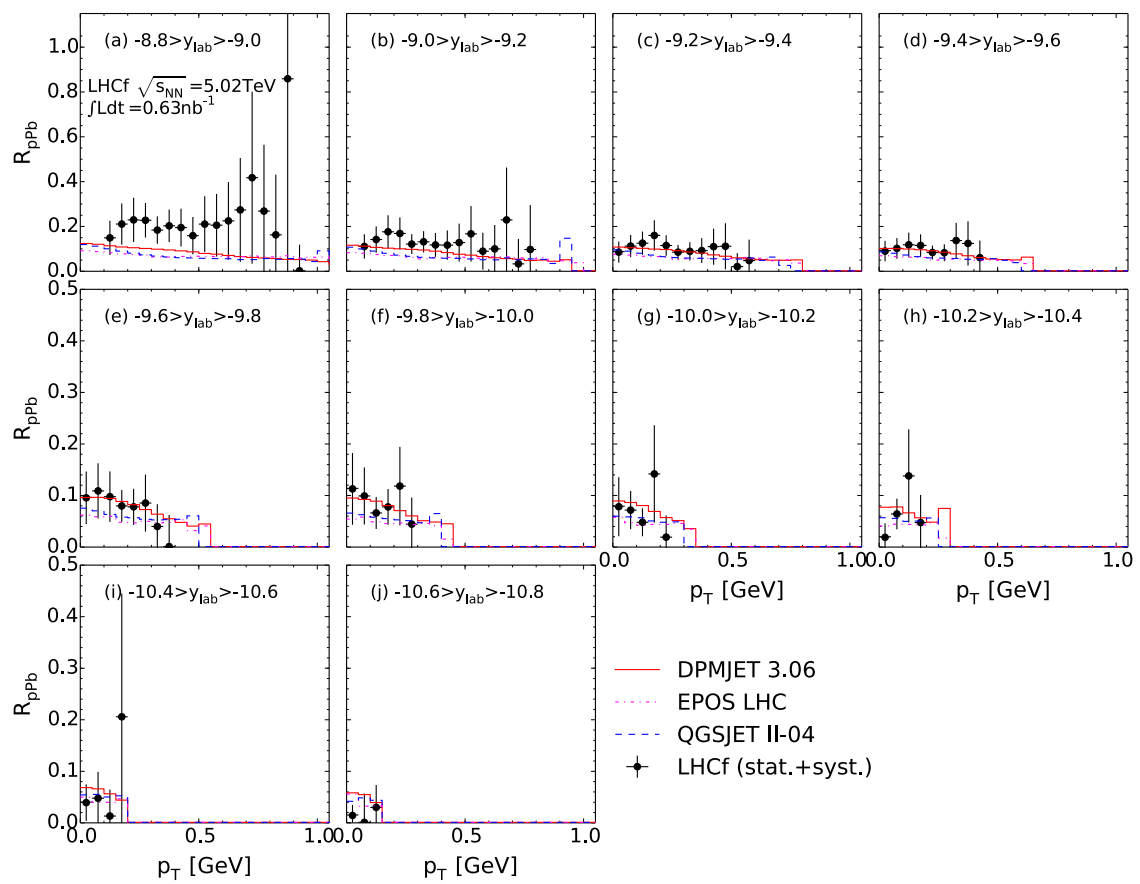

Figure 6. Nuclear modification factor for neutral pions in $\mathrm{p}-\mathrm{Pb}$ collisions at $5.02 \mathrm{TeV}$ : coloured histograms show results from Monte Carlo simulations with different hadronic interaction models, experimental data are shown as black points.

\subsubsection{Test of Feynman scaling}

The Feynman scaling rule [20] states that the production cross sections of secondary particles as a function of the Feynman-x variable $\left(X_{F} \equiv 2 P_{Z} / \sqrt{s}\right)$ were independent of the incident energy in the forward region. If the Feynman scaling holds, the differential cross section $\left(X_{F} / \sigma_{\text {inel }}\right)\left(d \sigma / d X_{F}\right)$ should be independent of the center of mass energy for $X_{F} \gtrsim 0.2$. 
This scaling rule was tested with the LHCf data at $\sqrt{s}=2.76 \mathrm{TeV}$ and $\sqrt{s}=7 \mathrm{TeV}$. The test was possible only in the $P_{T}$ range $0.0 \mathrm{GeV}<P_{T}<0.4 \mathrm{GeV}$ because there are no data at $2.76 \mathrm{TeV}$ outside this range. The $X_{F}$ distributions of data at $7 \mathrm{TeV}$ and $2.76 \mathrm{TeV}$ are shown in figure 7 for two different $P_{T}$ ranges. The comparison of the two data sample indicates that the Feynman scaling holds at the $\sim 20 \%$ level.

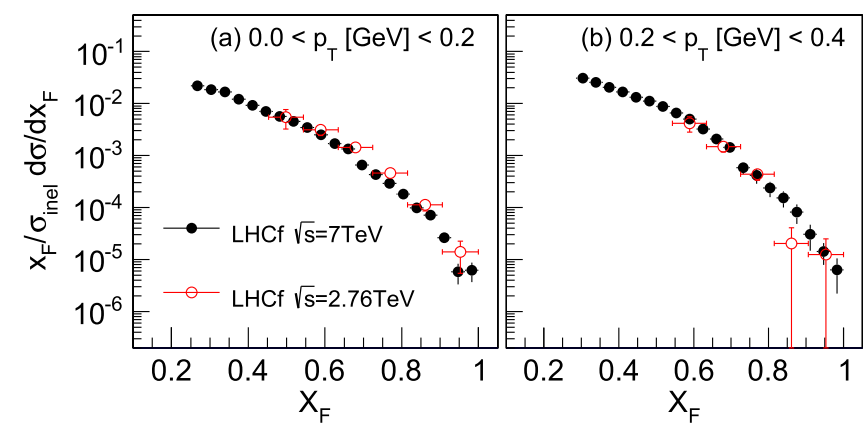

Figure 7. $X_{F}$ distributions of LHCf data at $\sqrt{s}=7 \mathrm{TeV}$ (black points) and $\sqrt{s}=2.76 \mathrm{TeV}$ (red points) in two $P_{T}$ ranges. The distributions are consistent at the $\sim 20 \%$ level.

\subsection{Photon inclusive energy spectra in p-p collisions at $\sqrt{s}=13 \mathrm{TeV}$}

Proton-proton collisions at $\sqrt{s}=13 \mathrm{TeV}$ were produced for the first time in 2015 at LHC. LHCf had a dedicated low-luminosity run from 9th to 13th of June 2015, with an instantaneously luminosity of $0.5 \div 1.6 \times 10^{29} \mathrm{~cm}^{-2} \mathrm{~s}^{-1}$. The preliminary analysis of photon spectrum was done on the data obtained during the LHC Fill \#3855. The integrated luminosity was $0.23 \mathrm{nb}^{-1}$ for both Arm1 and Arm2. The preliminary inclusive energy spectra of photon produced in p-p collisions at $\sqrt{s}=13 \mathrm{TeV}$ are presented in figure 8 for two pseudo-rapidity ranges together with the predictions of QGSJET, EPOS, SIBYLL, DPMJET and PYTHIA hadronic interaction models. The LHCf data lie between MC predictions but there is not an unique model with a good agreement in the whole energy range.

\section{Conclusions}

LHCf experiment performed measurements on forward production of neutral particles in protonproton and proton-lead collisions at LHC accelerator. These measurements are necessary to calibrate hadronic interaction models used in cosmic rays physics to understand the development of atmospheric showers. LHCf performed measurements at $\sqrt{s}=0.9,2.76,7$ and $13 \mathrm{TeV}$ in p-p collisions and at $\sqrt{s_{N N}}=5.02 \mathrm{TeV}$ in $\mathrm{p}-\mathrm{Pb}$ collisions.

In this paper latest published results about $\pi^{0}$ analysis (p-p at 2.76 and $7 \mathrm{TeV}, \mathrm{p}-\mathrm{Pb}$ at $5.02 \mathrm{TeV}$ ) and neutron analysis ( $\mathrm{p}-\mathrm{p}$ at $7 \mathrm{TeV}$ ) were presented. Preliminary results from neutron analysis indicate a large amount of high-energy hadrons in the very forward region, predicted only by QGSJETII-03 model. $\pi^{0} P_{T}$ and $P_{Z}$ measured spectra show a better agreement with QGSJET II-04 (in the whole range) and EPOS-LHC (for $P_{T}<0.5 \mathrm{GeV}$ and $P_{Z}<2 \mathrm{TeV}$ ). $\pi^{0}$ analysis in p-Pb indicates a strong suppression of $\pi^{0}$ production compared to $\mathrm{p}$-p case; nuclear modification factor from data is consistent with MC models predictions within errors. Feynman scaling hypotesis was tested comparing $\pi^{0}$ spectra at 7 and $2.76 \mathrm{TeV}$; the results indicate that the rule holds at the $\sim 20 \%$ level. Preliminary photon energy spectra indicate that there is not an unique model representing the data, but the measured data lie between MC predictions. 

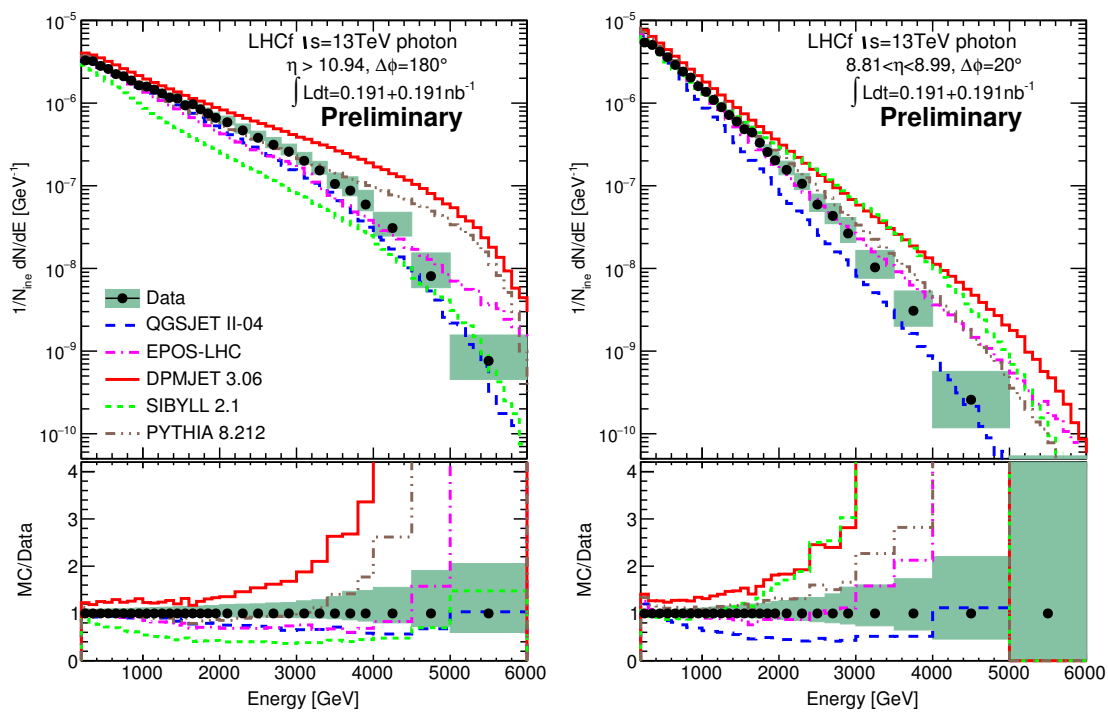

Figure 8. Preliminary inclusive photon energy spectrum for pseudorapidity $\eta>10.94$ (left figure) and $8.81<$ $\eta<8.99$ (right picture). Data are represented by black point while MC prediction from several models are represented by coloured histograms. Green shaded area represents statistical+systematic errors of data.

\section{References}

[1] O. Adriani et al., JINST 3, S08006 (2008).

[2] O. Adriani et al., JINST 5, P01012 (2010).

[3] K. Kawade et al., JINST 9, P03016 (2014).

[4] O. Adriani et al., Physics Letters B 715, 298-303 (2012).

[5] O. Adriani et al., Physics Letters B 703, 128-134 (2011).

[6] O. Adriani et al., Physical Review D 86, 092001 (2012).

[7] O. Adriani et al., Physical Review C 89, 065209 (2014).

[8] O. Adriani et al., Physics Letters B 750, 360-366 (2015).

[9] O. Adriani et al., Physical Review D 94, 032007 (2016).

[10] K. Taki et al., JINST 7, T01003 (2012).

[11] G. D’ Agostini, Nucl. Instrum. Meth. A 362, 487 (1995).

[12] S. Ostapchenko, Nucl. Phys. B, Proc. Suppl. 151, 143 (2006).

[13] K. Werner, F.-M. Liu, and T. Pierog, Phys. Rev. C 74, 044902 (2006).

[14] V. Gribov, Sov. Phys. J. Exp. Theor. Phys. 26, 414 (1968).

[15] T. Regge, Nuovo Cimento 14, 951 (1959

[16] E.-J. Ahn, R. Engel, T. K. Gaisser, P. Lipari, and T. Stanev, Phys. Rev. D 80, 094003 (2009).

[17] F. W. Bopp, J. Ranft, R. Engel, and S. Roesler, Phys. Rev. C 77, 014904 (2008); R. Engel, J.

Ranft, and S. Roesler, Phys. Rev. D 55, 6957 (1997).

[18] T. Sjöstand, S. Mrenna, and P. Skands, J. High Energy Phys. 05 (2006) 026.

[19] T. Sjöstand, S. Mrenna, and P. Skands, Comput. Phys. Commun. 178, 852 (2008).).

[20] R. P. Feynman, Physical Review Letters 23, 1415 (1969). 